\title{
The Model of Appropriation
}

\author{
Contribution of Rational Choice Theory and Cognitive Science to a Better Technology
}

\author{
David Zejda \\ Faculty of Informatics and Management \\ University of Hradec Králové \\ Hradec Králové, Czech Republic \\ e-mail: david.zejda@uhk.cz
}

\begin{abstract}
Ambient intelligence, as well as other fields of advanced applied science and technology, has a potential to improve quality of our lives. But the whole context, including psychological, and social aspects, have to be considered if we wish to make our technology resonating with user's real needs. The paper shows, how may rational choice theory and cognitive science contribute to describe and understand the mental processes which occupy user's mind during the appropriation stage of technology evaluation. As a main contribution, we present a simple model of appropriation, both verbally and in a semi-formal numerical form. Even though we are focused primarily on ambient intelligence, the model is generic enough to be applied in other fields too. The model implies, besides else, that enthusiasm induced by an early evaluation does not necessarily raise chances of success in the following appropriation stage.
\end{abstract}

Appropriation; rational choice theory; cognitive science; technology evaluation; ambient intelligence

\section{INTRODUCTION}

Will be a certain product or a technology accepted by users? Which factors play a significant role? How to design and develop products which will be widely accepted by a certain target group? How to define the best target group for the intelligent product we developed? How to accommodate an user interface for a target group? How to introduce our product to users? To be able to deliver really beneficial products, we should know how to answer the questions. To find the way, we have to transcend the field of ambient intelligence and the technology itself, bring results of research from different fields of science, and fit them together.

Though we are still a bit lacking the interdisciplinary extent of research, the idea is not totally new, of course. For example, Kamal Jit Singh mentioned in his plenary talk at the beginning of IE'10 conference [1] that technology should not be viewed as a silver bullet - people from all corners of the world may benefit from advancement in technology, including ambient intelligence, but if our aim is to truly improve their lives, broader context, including psychological, sociological and cultural implications, has to be considered. Also Mario Tokoro in his proposal of "open systems science" emphasizes the interdisciplinarity as a key aspect of science of the future, the science able to deal with the big, global and open issues of the present and the following days. [2]
Back to the acceptability of ambient intelligence - we have to find out, how users think about products and technologies, how they evaluate, handle, apply, and which aspects do they consider and which other circumstances play a role in these processes. Initial stage of a process of technology evaluation leads to a decision whether to give a product try or not. Appropriation is the stage of evaluation which follows upon successful acceptance. We may say that a product has been finally appropriated if it has become integral part of user's daily activities, failing which the product is rejected, e.g. if the product does not fulfil user's initial expectations well.

The semi-formal model introduced in this paper brings insight into the process of appropriation. Though intentionally simplified, the model may assist researchers and designers to keep on mind these psychological aspects while working on technological concepts or turning them into products. It may also help those who are responsible for introducing novel products and technologies to prospective users to select the strategy, which will likely lead to the best results in terms of products successfully appropriated by their users and low counts of those who are disappointed.

We build primarily upon research in ambient intelligence, economics (rational choice in particular), and cognitive science subfield of psychology, the current leading descriptive conceptualization of processes within human minds. Besides the model as a synthesis of research in all the fields, as a secondary contribution of the paper we present surfaces of contact between these fields of science. Rational choice theory brings precise tools of economy to describe our reasoning in great variety of life situations. But, we as human beings are not rationally reasoning machines, we do not think, recollect, decide, or behave precisely, logically and deterministically. Actually our mind brings more biases and less rationality than what we would like to believe. Rational choice theory brings good answers on the question how we should think and behave (its nature is naturally normative), whereas cognitive science is much more descriptive, aiming to capture how we indeed think and behave, thus reflects many of the inconsistencies, biases, flaws of the reasoning machines in our heads.

Both rational choice theory and cognitive science research already concluded in various useful conceptual models. Because processes in human mind are highly complex, every model attempting to describe them has to apply various simplifications, which means also that the approximate results from all the models have to be applied with caution. 


\section{Areas of Related Research}

We may think of appropriation from various perspectives, stressing technical, sociological, psychological, or e.g. medical aspects. Each of the approaches may contribute to our understanding. As mentioned in the introduction, we decided to rely primarily on two conceptual frames, the rational choice and cognitive science.

\section{A. Rational Choice}

Rational choice view brings formality to our consideration, because as a descendant or a subfield of economy it disposes of great palette of diverse formal models. Gary Becker, well known proponent of the approach, used rational choice theory means to describe many aspects of human behaviour [3] including drug addiction [4], beggary and compassion [5], discrimination [6], crime and punishment [7], human capital [8 ], love, marriage and family [9] and according to him, nearly all human behaviour may be explained as rational reasoning. Rationality involves balancing costs against benefits in order to maximize advantage, where the advantage is not necessarily defined in terms of money. [10]

On the conception of rational theory we already built and published a simple model of technology evaluation, with specific focus on ambient intelligence for ageing users. [11] The model describes first stage of technology evaluation, when a prospective user is thinking whether to try certain product or not. In compliance with other rational choice models (e.g. Bentham [12] or Marshall [13]), the advantage in the model is not defined in the monetary expression, but rather emphasizing more subtle determinants. In the case of the model of evaluation, the advantage reflects specific needs of the considered user group of the elderly, such as perceived selfworthiness and social relations. Personal utility function assigns utility value to each combination of the comfort sources. Comfort sources are fully determined by the complete set of influence vectors, where each vector is assigned to certain aspect of life. Instead of monetary expressed cost, the model comprises effort, time and external support as resources. The exert function defines how much resources are necessary to adopt an aspect of life.

The model describes initial evaluation as a sequence of three successive steps, leading to either acceptance or one of three possible kinds of refusal. If an aspect of life (e.g. product) is perceived as 1 . to be beneficial, 2. to be reachable and 3. the best choice from all available options, it passes the evaluation stage and may proceed to the appropriation phase. The simple evaluation model may be solved as an optimization problem of linear programming.[14]

This paper concurs the evaluation model, because where evaluation stage concludes (user decides to give a product try), appropriation stage follows - see I. and II. on Fig. 1. In the appropriation stage, the product has to become integrated into user's daily behaviour. Though we take rational choice and also the evaluation model mentioned above into account for our further reasoning, we decided to rely primarily on the cognitive science. The main reason is, that we are not as much rational as we would like to believe. Cognitive science revealed, that there are flaws in our reasoning even in simple isolated tasks, as described further. Many more such flaws, biases, irrationalities influence our reasoning in the tasks which are more difficult and scattered in a longer period of time. So, if rational choice approach was satisfying for comparatively simple evaluation task, more complex appropriation requires to pay more attention to our irrationality.

\section{B. Cognitive Science}

Psychology developed significantly during the last century. Where former theories such as Freudian psychoanalysis or behaviourism failed to explain various phenomena in our reasoning, cognitive science, based on precise scientific methods including rigorous statistical examinations in reproducible scenarios, markedly deepened our understanding of ourselves. [15] The appropriation model introduced later in the paper, though drawing from rational choice theory and tied together with the rational theory model of technology evaluation, takes cognitive science as an essential basis.

From all concepts revealed by the cognitive science some appeared as very relevant to our problem. We considered them thoroughly and we reflected most of them directly in the model. Some of the concepts are also further discussed in the final section on possible model refinements and extensions.

\section{1) Substages of Evaluation}

A lot of current scientific understanding of human reasoning comes from studies of behaviour in highly controlled simple tasks, where participants decide between well-defined options, such as single gambles. Real world situations are both not so much controllable, and also more complex, requiring sequential evaluation. Appropriation is a complex and dynamic task. Actually, explanation of dynamic tasks, chains of successive related decisions, is one of the current leading topics in the cognitive science. Beginning of this trend may be traced to work of Damasio et al. and their study of the performance of brain-injured patients. [16] Many researchers followed the path, e.g. Lejuez et al. [17] examined, how participants think and behave in balloon inflating task.

As an conclusion from these studies we may say, that it is quite natural to human mind to break a complex problem into distinctive steps, framed according to the character of the task (such as single blows in the balloon experiment). The choice process is often distributed across an interval of time and composed of choice episodes or steps. [18]

\section{2) Anchors or Reference Points}

Another concept very well described in many studies is the tendency of our mind to focus on certain fact for further reasoning. Kahneman and Tversky [19] (among others) revealed initially in monetary decisions the phenomenon of anchor (or reference point). Instead of reasoning in absolute quantities and final outcomes, our mind tends to think in relative comparisons and in shorter time frames. Both positive and negative outcomes of our decisions have diminishing returns proportionally to the distance from the reference point.

Though initially introduced by an economist, the concept of labile, vague, adaptive reference points which is not always rational has never been incorporated in the mainstream economy, showing its primary focus on normative description of human reasoning. Relative approach of our minds, though tenable in certain situations, when applied in tasks with utility, contradicts with traditional diminishing marginal utility law known in economy, and rational choice theory as well. With 
these findings Kahneman and Tversky refined the theory of utility under a new term, the prospect theory.

As further research revealed, palette of various reference anchors exist, either related to the problem, such as status quo or aspiration level, or totally irrelevant, as proven e.g. in [20]. Some of them are more prevalent and more influential, such as status quo. Lopes and Oden [21] concluded that at least three reference points play significant role in our evaluations under uncertainty. They act in parallel: main reference point (usually status quo), aspiration level, and security level (danger of loss). According to Hastie and Dawes [18] the model built around the concept of reference points may compete with prospect theory with its ability to describe our reasoning.

\section{3) Gradual Adaptation}

The concept of gradual adjustment is nothing more than an application of the concept of mental anchors in complex problems with successive decision chains. Concrete examples of this phenomenon are mentioned e.g. in [18]. When we respond to stimuli, such as loudness or temperature, the past and present context of experience defines an adaptation level, or reference point, and stimuli are perceived in relation to this point. Cyert and March revealed, that we tend to search for alternatives in the neighbourhood of our previous try. [22]

In complex schemes with successive steps, we tend to follow the anchor-and-adjust strategy, which leads to successive adjustment on-the-fly. E.g. Slovic et al. confirmed the effect in pricing and choice in successive virtual gambles. [23] Kahneman and Tversky pointed out, that justification for reasoning on consequences with status quo on mind can be found in the general principle of adaptation - the stepwise adjustment of the mind anchor allows to adopt our mind to the always changing environment. On the other hand it may easily lead to illogical flaws in our reasoning, such as "money pump" described in [18], which contradicts with rational (economic) choices.

\section{4) Linear Model}

According to many studies, if the task is to make a decision based on a set of cues, we could hardly name anything better to describe our reasoning, than a very simple linear model. Actually, even improper linear models with weights not based on statistical techniques (e.g. with random weights, where only direction of relation is explicitly assigned) outperform experts in many expert tasks [24].

Yes, though it may be hard to believe, simple linear model executed by dumb computer program achieves same or better results in medical diagnosis tasks [25], in prediction of bankruptcy [26], in assessment of applicants [27], in estimations of real estates values, in stock investments and many other areas. Since first notable book on the topic has been published more than 50 years ago [28], huge amount of studies concluded again the same. So, nowadays there is no controversy on this general inability in reasoning - any "expert insight" is more than outweighed with inconsistency, incomplete memory, and plenty of other flaws. According to March [18], the fact that we still rely on experts despite their real incompetence may serve a purely social function. With all these findings, if we wish to formally describe our reasoning in situations based on a set of cues, linear equation is pretty much sufficient, no need to seek for anything more complicated (more rational).

\section{5) Two Chains of Reasoning}

Interesting and currently "hot" subfield of cognitive science is neuroscience (called also neuroeconomics). Researchers in this area examine neural substrates of our judgment and behaviour. [18] One of conclusions made by neuroscience is the fact, that our reasoning runs over internally in two trails (chains, circuits). While dopamine-mediated system is responsible for assessing positivity, acetylcholine-mediated circuit ensures negativity. From the works we may mention e.g. [29].

What is relevant to our model, any evaluation runs over in parallel. User evaluates benefits, utility, rejoice, pleasurable surprise etc. on one hand and negative aspects such as costs, pain, anger, disappointment on the other hand at the same time.

\section{The Model of Appropriation}

In the previous section we already mentioned, that the stage of appropriation logically follows previous stage, an initial evaluation or the acceptance. So, as a starting point for appropriation we have a product and a person who decided to give the product a try. While the model of evaluation emphasizes variety of aspects of life (e.g. products) and associated anticipated benefits and costs, in the appropriation phase we hold much closer look on the one product which passed evaluation. To keep the model simple, we abstract from variety of other alternatives where to put effort and how to spend time. Our model is descriptive, not normative - we do not wish to advice how to perform the appropriation rationally, rather we wish to describe the reasoning as it usually takes place in minds of users in real.

\section{A. The Motivation and Resources}

A user starts the appropriation with certain expectations, described in the model of evaluation as the anticipated influence of the product on aspects of his life, which would be perceived as a benefit (utility). The expectations play role during the whole appropriation process, though successively adjusted by the anchor-and-adjust strategy. The same expectations also consist base for initial enthusiasm ${ }^{1}$, which turns into patience with the product and plays significant role as well, as described below. Only if the user keeps his positive motivation during the whole process, product may be appropriated. Various forces influence the motivation:

Initial component. First of them is an initial component, comprising expectations and the initial enthusiasm lowered by initial qualms ("Yes, it will be great to keep in touch with my close daily with that machine tool.. Hmm, but looks a bit difficult to grasp meanings of the buttons.."). Enthusiasm usually falls since the beginning, which is induced by two factors in the model, the nearly inevitable bore and much more avoidable disappointment.

External component. Second, motivation may be supported by external forces throughout the process ("My dear little looks really enthusiastic, she keeps telling me how great is the weird looking apparatus.. Hmm, not sure, but I do not wish to disappoint her.”). Excessive external encouragements may be

\footnotetext{
${ }^{1}$ The mechanisms how expectations turn into enthusiasm and patience are out of border of the presented model, we are more focused on the description of successive steps which follow.
} 
counter-productive. We decided to apply a strategy common in the economics and remove this particular dimension of the problem, because it could distract attention from the core ideas of the appropriation process. This way the model will remain simple and external motivation component may be incorporated in its later extension.

Habitual component. Last and steadiest source of motivation is something what we call habitual component. Only the technologies which break into daily patterns of behaviour may survive in long term. The product has to prove clear and significant benefits for life during the appropriation. The idea is reflected in gradually rising perceived fulfillment of expected utility and gradual rise of "the mastering", the ability to use the technology and use it effectively. Habitual component grows gradually if the user gradually finds out that there is nothing to fear from and the technology fulfils his expectations and slowly integrates into his of his daily life. It may grow even suddenly, when the technology exceeds expectations, leading to positive surprise. The situation is reflected as a disappointment with negative sign in the model.

Economy and also rational reasoning in general is about effective management with limited resources. Cognitive science does not presume full rationality, but the idea of weighing benefits and resources appears there as well, though not in so formal shape, reflecting various irrational flaws in our reasoning, e.g. [19]. In the appropriation process as described in our model, certain aspects play the role of constraints too the effort which has to be expended to manage the product has to fit within borders of reachability, also the patience inevitable diminished by bore set up deadline for the product to be successfully appropriated.

\section{B. Substages of the Model}

In compliance with "substages" principle, we treat the appropriation as a process which consists of a chain of successive recurring evaluation steps. Each step may lead in either one of final solutions (appropriation, rejection) or in continuation of the process. Though the model is described regardless to the duration of one step, we assume that the most suitable candidate is one day, bearing on mind that appropriation usually requires several or even many days. Day is definitely one of the time frames we are used to think in, maybe the one most common. Further research would be necessary to confirm the assumption.

Informal flowchart on the picture 1 illustrates the single appropriation step schematically. As shown in the picture, the initial evaluation (or acceptance) stage I. precedes II., which is the stage of appropriation examined in this paper. Each appropriation step has its own structure and fall into several substeps. Numbers in the chart denote the individual substeps described below.

1. Expectation: At the beginning of each step, user has certain expectations of the effort which has to be expended and the corresponding outcome (growth in mastering and achieved utility).

2. Exertion: He has to expend certain amount of effort during the step - to think of the product, to learn how to handle it a bit, to try, to try again. The effort as used in the model comprises both intellectual or manual strain and the time necessary. For simplicity, as explained above, we do not assume other resources, such as external support.

Figure 1. Schematic illustration of the appropriation process

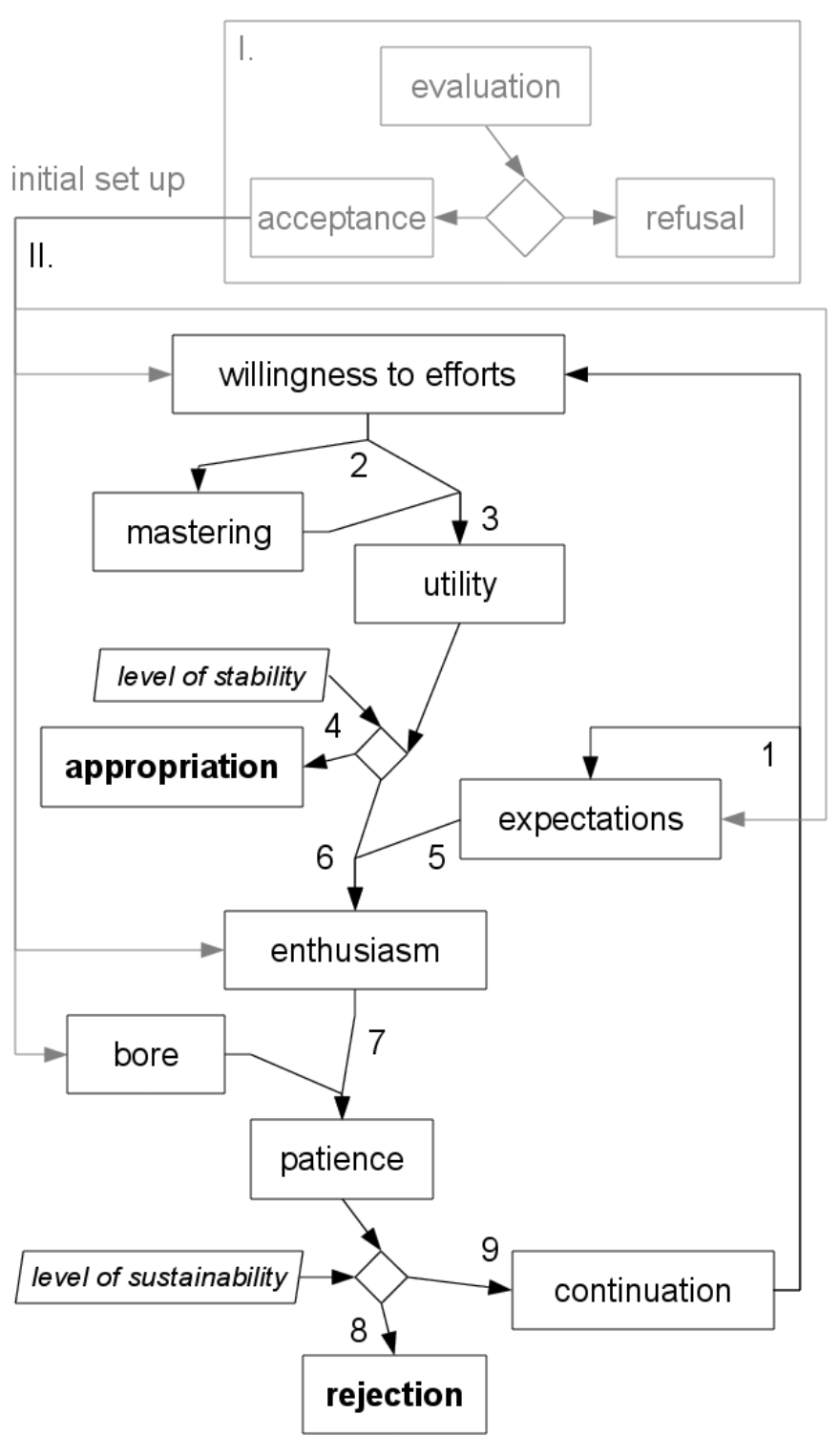

3. Growth: The expenditure during exertion has two primary effects. First, the level of mastering grows user learned how to use the product a bit or get used to use it a bit. Second, the level of the perceived utility ${ }^{2}$ grows as well, as the user see positive impacts on his well-being. The growth in mastering may turn into an additional contribution to the utility.

4. Appropriation: If the growth of utility induced by exertion in the step reaches certain value which we call the level of stability, the product has been finally appropriated. Level of stability may be reached

\footnotetext{
${ }^{2}$ Yes, we call both expected and achieved benefit in our model "utility", even though some of cognitive scientists prefer other terms, such as personal value. [18] We decided so, because term utility is much more common, more understandable for greater audience and also to be consistent with the former model of evaluation.
} 
regardless to the level of mastering. Since the moment of successful appropriation, patience is no more necessary to overcome bore and the whole appropriation process ends. Level of stability, though not fully equivalent, closely matches with the aspiration reference point of final expectations (see above, the section on anchors or [21] [20]). The level is primarily determined in the evaluation phase, is not fully rational and may be influenced even by irrelevant factors. For simplicity we abstract from its further adjustments during appropriation.

5. Comparison: User already knows which amount of effort he had to expend and what were the outcomes of the step, so now he compares the reality with his initial expectations. Each of the components (effort necessary, progress in mastering, achieved utility) may bring either disappointment or positive surprise.

6. Assessment: If the overall result of comparison phase is a positive surprise, it positively influences his enthusiasm and vice versa.

7. Affection: Enthusiasm induced by positive surprise (or drop in enthusiasm induced by disappointment) affects patience. Another force, called the bore, causes that patience gradually diminishes step by step, regardless to immediate surprise/disappointment effects.

8. Rejection: If remaining patience drops below certain value which we call the level of sustainability, the product is being rejected ${ }^{3}$ and the whole appropriation process ends. The level of sustainability may be reached regardless to the level of mastering or level of perceived utility. Level of sustainability, similarly to the level of stability, is primarily determined in the evaluation phase, as a result of reasoning on prospective final utility in the context of the whole appropriation process. Actually, we may view the level of sustainability as an incarnation of security reference point. [21] Again, for simplicity we abstract from further adjustments during the process.

9. Continuation: Appropriation follows into next step, with updated level of patience and also, in compliance with anchor-and-adjust strategy, with updated expected necessary effort and updated expected outcomes.

\section{Semi-formal Numeric Representation}

The aim of this section is not to bring precise highly formal representation of the model, but rather use numerical representation to outline the idea better. Unlike to as defined above, in this section we further abstract from enthusiasm. Initial enthusiasm derived from evaluation phase goes in the model directly as patience. The chain disappointment $\rightarrow$ enthusiasm $\rightarrow$ patience has been degraded to simpler disappointment $\rightarrow$ patience.

So, first, let's name basic cumulative variables, measured on the percent scale $0 . .100 \%$ :

\footnotetext{
${ }^{3}$ The term rejection has been selected to avoid confusion with "refusal" as defined in the evaluation model and we consistently distinguish the two terms in the paper.
}

- $\mathrm{Me}_{\mathrm{n}}$ : level of mastering which is expected to be reached in the $n$-th step

- $\mathrm{Mr}_{\mathrm{n}}$ : level of mastering which has been reached in the n-th step

- $\mathrm{Ue}_{\mathrm{n}}$ : level of utility expected from the technology in the $n$-th step

- $\mathrm{Ur}_{\mathrm{n}}$ : level of utility reached from the technology in the n-th step

- $\quad \mathrm{P}_{\mathrm{n}}$ : level of patience with the technology reached in the $n$-th step

Variables representing effort necessary for each step and disappointment induced by the step:

- $E_{\mathrm{n}}$ : effort which is expected to be necessary for the n-th step

- $\mathrm{Er}_{\mathrm{n}}$ : effort which was necessary for the n-th step

- $\mathrm{D}_{\mathrm{n}}$ : disappointment with the technology induced by the $\mathrm{n}$-th step

Two constants representing aspiration and security level (discussed above):

- $\mathrm{u}_{\mathrm{s}}$ : level of stability

- $\quad \mathrm{p}_{\mathrm{s}}$ : level of sustainability

And several constants which reflect subjective character of the user, his tendency to weariness and perception of surprise/disappointment:

- $\quad$ b: bore with the technology induced in a step

- $\mathrm{S}_{\mathrm{d}}$ : significance of underestimation of effort

- $\mathrm{s}_{\mathrm{m}}$ : significance of underest. of mastering increase

- $\mathrm{s}_{\mathrm{u}}$ : significance of underest. of utility increase

The preceding evaluation phase concludes in:

1. Initial expectations of necessary effort $\mathrm{Ee}_{1}$, mastering after the first step $\mathrm{Me}_{1}$, utility after the first step $\mathrm{Ue}_{1}{ }^{4}$

2. Initial level of enthusiasm turned into initial patience level $\mathrm{P}_{1}$.

3. Bore $b$, level of stability $u_{s}$ and level of sustainability $\mathrm{p}_{\mathrm{s}}$, all of them remain the same during the whole appropriation process. ${ }^{5}$

So, evaluation phase brings initial expectations $\mathrm{Ee}_{1}, \mathrm{Me}_{1}$, $\mathrm{Ue}_{1}$ to the first step of appropriation.

At the beginning of the second step the expected effort is the same as the effort required in the first step:

$$
\mathrm{Ee}_{2}=\mathrm{Er}_{1}
$$

\footnotetext{
${ }^{4}$ Assuming linear increase as a simplest case, $\mathrm{Me}_{1}$ and $\mathrm{Ue}_{1}$ may be defined as $100 \%$ divided by expected length of appropriation.

${ }^{5}$ Dynamically adapting variables instead of constants are suggested in the later section as a possible model refinement.
} 
Similarly, expected rise in mastering and utility induced by the second step is determined by the levels achieved in the first step.

Because $M$ and $U$ are cumulative quantities the level expected at the end of second step is double:

$$
\begin{aligned}
\mathrm{Me}_{2} & =2 \mathrm{Mr}_{1} \\
\mathrm{Ue}_{2} & =2 \mathrm{Ur}_{1}
\end{aligned}
$$

And at the beginning of $n$-th step other than first or second the expectations are again determined by the results in the previous (n-1-th) step:

$$
\begin{aligned}
& \mathrm{Ee}_{\mathrm{n}}=\mathrm{Er}_{\mathrm{n}-1} \\
& \mathrm{Me}_{\mathrm{n}}=\mathrm{Mr}_{\mathrm{n}-1}+\left(\mathrm{Mr}_{\mathrm{n}-1}-\mathrm{Mr}_{\mathrm{n}-2}\right)=2 \mathrm{Mr}_{\mathrm{n}-1}-\mathrm{Mr}_{\mathrm{n}-2} \\
& \mathrm{Ue}_{\mathrm{n}}=\mathrm{Ur}_{\mathrm{n}-1}+\left(\mathrm{Ur}_{\mathrm{n}-1}-\mathrm{Ur}_{\mathrm{n}-2}\right)=2 \mathrm{Ur}_{\mathrm{n}-1}-\mathrm{Ur}_{\mathrm{n}-2}
\end{aligned}
$$

At the end of any step $n$ we know real values of $\mathrm{Er}_{\mathrm{n}}, \mathrm{Mr}_{\mathrm{n}}$, $\mathrm{Ur}_{\mathrm{n}}$, so comparison against the expectations may take place:

If $\mathrm{Ur}_{\mathrm{n}}$ reaches $\mathrm{u}_{\mathrm{s}}$, the step results in appropriation.

$\mathrm{D}_{\mathrm{n}}=\mathrm{s}_{\mathrm{d}}\left(\mathrm{Er}_{\mathrm{n}}-\mathrm{Ee}_{\mathrm{n}}\right)-\mathrm{s}_{\mathrm{m}}\left(\mathrm{Mr}_{\mathrm{n}}-\mathrm{Me}_{\mathrm{n}}\right)-\mathrm{s}_{\mathrm{u}}\left(\mathrm{Ur}_{\mathrm{n}}-\mathrm{Me}_{\mathrm{n}}\right)$

$\mathrm{P}_{\mathrm{n}}=\mathrm{P}_{\mathrm{n}-1}-\mathrm{b}-\mathrm{D}_{\mathrm{n}}$

If $\mathrm{P}_{\mathrm{n}}$ drops below $\mathrm{p}_{\mathrm{s}}$, the step results in rejection.

\section{Implications for Design Process}

We may think about various ways how to make our products more "appropriable" and there are various implications both from cognitive science and from the model itself. The model suggests, that there may be various causes of rejection. In general, product is rejected, whenever user runs out of patience before the level of stability is reached. It may happen because of lack of initial enthusiasm, because of boring activity related to the product mastering, or because of disappointment when the product does not satisfy user's aspiration level reflected in expectations.

Certain qualities defined in the model describe personal psychological characteristics of users, such as significances of underestimation. Other qualities may be influenced, but only partly because their subjective component is significant, such as bore induced by every step of evaluation. The bore may be slightly decreased if we e.g. lower demands on effort or make the effort more pleasurable. Some variables are in great degree in hands of designers, such as effort really necessary for the appropriation and the real beneficial potential of the product. Other variables, such as enthusiasm, expectations, and levels of sustainability and stability, are at least partially in hands of those who introduce the product to the users. The variables may be influenced in many ways, e.g. through both relevant and irrelevant aspects of the situation in which we introduce the product. As an example, let's discuss enthusiasm in a more detail:

\section{A. Balance The Induced Enthusiasm Carefully}

Enthusiasm on one hand induces higher level of patience, which is good, because sufficient "supply of patience" induced primarily from enthusiasm is necessary to overcome bore gradually induced in each step. But it is primarily determined from expectations and with higher expectations there is higher chance of disappointment, on equal terms (which is bad). It means, that higher initial enthusiasm does not necessarily mean higher success rate (or lower probability of rejection) in appropriation, as illustrated on picture 2 .

Though explanations of reasons behind this effect differ, there is consensus among economists and cognitive scientists, that loss is more painful than gain. It indicates that disappointment should be avoided if our goal is higher chance of successful appropriation. Our goal could be to induce the highest level of enthusiasm, which will (hopefully) not lead to disappointment.

Figure 2. The two chains of influence of expectations on patience

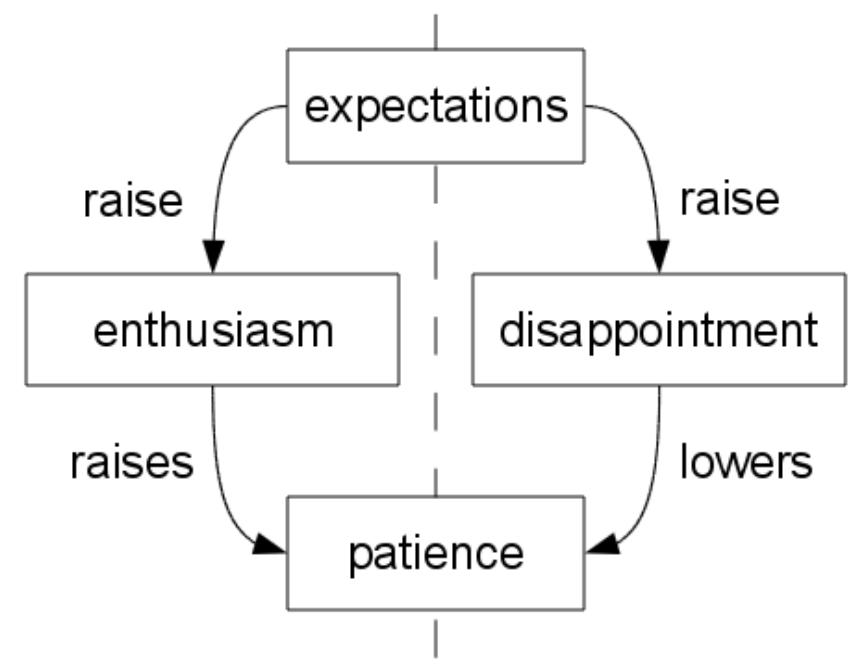

But, this maximal level of enthusiasm not inducing disappointment may be still too high. Mellers et al. arranged specific gambling task to capture regret and rejoice reactions and concluded, that experienced utility is intensified if it produces regret or rejoicing, in particular if it is a surprise. [30] Our model allows negative value of disappointment, which may be interpreted as opposite of disappointment, a positive surprise. The exact effects of surprise on our perception of utility are still not fully examined. According to [18] it is still not clear under which conditions people actually infer and consider counterfactual at the time they make the decision, that is, under which conditions the regret and rejoicing effects affect perception of utility and the process itself, but it seems, that in compliance with Mellers' conclusions, it may be better to undervalue the benefits of introduced product slightly. Lower level of enthusiasm (inducing patience) may be more than compensated with the intensified effects of positive surprise, leading to higher probability of successful appropriation.

One more reason to avoid inducing too high enthusiasm is our general tendency to overvalue impacts of our decisions on our well-being or happiness, both positive and negative. [31], [18] It is likely, though not fully examined yet, that this bias is slightly compensated with past-adjusting defensive strategy, according to which we reshape our memories to fit well to our perception of presence. Fischhoff demonstrated that people 
who know present events falsely overestimate their accuracy in which they would have predicted them. [32] Though hindsight contributes to diminish impact of expectations which fell short, it does not change the fact that the optimal level of enthusiasm is probably somewhere below the level not inducing disappointment.

\section{Possible Model Refinements and Extensions}

The model has been designed to match with most notable and relevant conclusions of cognitive science, but as very simple and only semi-formal. First of all, it should be formulated more precisely, preferably around a well defined set of axioms. Particular shapes of the model functions should be defined in this stage. The more formal axiomatized form of the model will allow evaluation of the model itself in a study with real users. The more formal form of the model will also allow application of additional mathematical tools to infer further predictions or conclusions. Certain aspects of the model could be refined or extended. Possible refinements include (but are not limited to):

- Disproportion in significance of outcomes, the fact that losses are in general more painful than gains are pleasant, is not only concluded in the theory of prospect, but also proven by further research and even economists agree, though it contradicts rationality. If we wish to reflect the fact in the model, we would have to redefine $D_{n}$, the disappointment with the technology induced by step $n$.

- $\quad$ Bore is defined in the model as constant, stable during the whole process. Actually, as anywhere else, it is highly probable that the anchor-and-adjust strategy applies here too, so the concept of bore could be refined to develop gradually during the steps of appropriation.

- We could distinguish between effort and time. Effort and time, together with external support, would frame set of mutually interchangeable resources - e.g. effort could be substituted with additional support etc. The resources could be defined either as objective (e.g. the time which the evaluator may invest), or as subjective (the time which is he willing to invest). Structured definition of resources would allow to apply additional concepts of economy to examine various implications. One of the concepts is the possibility frontier, describing combinations of resource levels allowing to continue with appropriation. Other is marginal rate of resource substitution, defined as the reduction in one resource input per unit increase in the other resource input that is just sufficient to maintain the product in appropriation, e.g. the marginal rate of substitution of time for support gives the amount of support that can be replaced by one unit of time. We could also examine changes in marginal rate of resource substitution between steps of evaluation and further implications.

- Concept of additional mental reference points reflected statically in the model as the level of stability and the level of sustainability may be refined to capture dynamics of anchor-and-adjust strategy.
- It is more natural to think in occurrences instead of probabilities or ratios [18], so $\mathrm{Me}_{\mathrm{n}}, \mathrm{Mr}_{\mathrm{n}}, \mathrm{Ue}_{\mathrm{n}}, \mathrm{Ur}_{\mathrm{n}}$ could be refined to reflect this fact if the aim would be to be as close as possible to our cognitive processes.

- Enthusiasm defined as scalar in the model could be unfolded to more structured description, reflecting at least positive and negative components (expected benefits and qualms).

- The whole model could be redefined from deterministic representation to probabilistic, to reflect uncertainty which definitely plays its role.

Possible extensions include (but are not limited to):

- Describe how expectations influence enthusiasm to give more concrete answer on the question which level of initial enthusiasm is optimal in regards to the chance of successful appropriation.

- The model abstracts from other opportunities fully, which is another simplification. Actually, e.g. level of stability could be defined as dependant on available alternative options of spending limited time.

- External supportive influence could be incorporated into model, comprising both external support and encouragements as a source of motivation. While encouragements add to the perceived utility and lowers level of stability, support lowers demands on effort and time spent by the user.

- Concept of "partial appropriation", where the technology is neither fully rejected, nor fully accepted, could be described.

Implications of other aspects of mental processes could be examined, such as dynamic inconsistency [33] (especially for long appropriation processes), previous either negative or positive experience as a basis of anchor for overall expectations, contrast with another (worse) product irrationally increasing perceived utility [20], influence of emotions evoked by external aspects of situation when the product is being introduced or when the appropriation steps occur [34], tendency to neglect tendency to regression toward average in feelings [31], or the tendency to neglect small changes (which could be projected in patience function as a resistance interval).

\section{Conclusions}

Both rational choice theory and cognitive science brings valuable insight into cognitive and mental processes, resulting in our cognition, and behaviour. Whereas rational choice theory brings more formal tools and great toolbox of concepts imported from economy and answers best on normative questions, cognitive science provides valuable much more descriptive view in our reasoning, which incorporates many of irrational aspects of our reasoning. Both fields of science may work together if we wish to understand, what is going on behind scenes in our minds in various situations. Based on the rational choice theory and even more on the cognitive science we created a simple model of reasoning which starts where a user already decided to give a product a try. The model has been described verbally, graphically illustrated and semiformalized as a set of equations. The model may tell, which 
powers in the stage of evaluation which we call appropriation makes the difference between final rejection or final adoption of the product which is being evaluated. Despite its simplification and generic nature, the model brings structured view on the appropriation process. As an example of interesting implication of the model we discussed the fact, that a high enthusiasm induced in the initial evaluation stage does not necessarily lead to a higher chance of success in the following appropriation stage.

\section{AcknOwledGMENT}

Supported by UHK FIM specific research no. 2110/2010 and by ESF grant INKOV reg. no. CZ.1.07/2.3.00/20.0001.

\section{REFERENCES}

[1] K. J. Singh, "Intelligent Environments '10 - Invited Speakers," 2010. [Online]. Available: http://intelligentenvironments.org [Accessed: 14-May-2011].

[2] M. Tokoro, "Open Systems Science: Solving Problems of Complex and Time-Varying Systems," 8th International Conference on Practical Applications of Agents and Multi-Agent Systems (PAAMS'10), 2010.

[3] G. S. Becker, The economic approach to human behavior. University of Chicago Press, 1976.

[4] G. S. Becker and K. M. Murphy, "A theory of rational addiction," The Journal of Political Economy, vol. 96, no. 4, 1988.

[5] G. S. Becker, Accounting for tastes. Harvard Univ Pr, 1998.

[6] G. S. Becker, The economics of discrimination. University of Chicago Press, 1971.

[7] G. S. Becker, "Crime and punishment: An economic approach," Journal of Political economy, vol. 76, no. 2, 1968.

[8] G. S. Becker and others, Human capital. National Bureau of economic research New York, 1975.

[9] G. S. Becker, A Treatise on the Family. Harvard Univ Pr, 1991.

[10] M. Friedman, Essays in positive economics. University of Chicago Press, 1953

[11] D. Zejda, "Deep Design for Ambient Intelligence: Toward Acceptable Appliances for Higher Quality of Life of the Elderly," in 2010 Sixth International Conference on Intelligent Environments, Kuala Lumpur, Malaysia, 2010, pp. 277-282.

[12] J. Bentham, Theory of legislation. Adamant Media Corporation, 1876.

[13] A. Marshall, "Principles of economics: an introductory volume," 1920.

[14] D. Zejda, "Ambient Intelligence Acceptable by the Elderly: Rational Choice Theory Model of Technology Evaluation for Deep Design," Proceedings of the Contexts Workshop of PAAMS conference 2011, 2011.

[15] B. J. Baars, The Cognitive Revolution in Psychology. Guilford Press, 1986.

[16] A. Bechara, H. Damasio, D. Tranel, and A. R. Damasio, "Deciding Advantageously Before Knowing the Advantageous Strategy," Science, vol. 275, no. 5304, pp. 1293 -1295, Feb. 1997.

[17] C. W. Lejuez, W. M. Aklin, M. J. Zvolensky, and C. M. Pedulla, "Evaluation of the Balloon Analogue Risk Task (BART) as a predictor of adolescent real-world risk-taking behaviours," Journal of Adolescence, vol. 26, no. 4, pp. 475-479, Aug. 2003.

[18] R. Hastie and R. M. Dawes, Rational Choice in an Uncertain World: The Psychology of Judgment and Decision Making. SAGE, 2009.

[19] D. Kahneman and A. Tversky, "Prospect theory: An analysis of decision under risk," Econometrica: Journal of the Econometric Society, p. 263291, 1979.

[20] A. Tversky and D. Kahneman, "Judgment under uncertainty: Heuristics and biases," Judgment and decision making: An interdisciplinary reader, p. 35, 2000.
[21] L. L. Lopes and G. C. Oden, "The role of aspiration level in risky choice: A comparison of cumulative prospect theory and SP/A theory," Journal of mathematical psychology, vol. 43, no. 2, p. 286-313, 1999.

[22] R. M. Cyert and J. G. March, A behavioral theory of the firm. Blackwell, 2005.

[23] P. Slovic, B. Fischhoff, and S. Lichtenstein, "Why study risk perception?," Risk analysis, vol. 2, no. 2, p. 83-93, 1982.

[24] R. M. Dawes, "The robust beauty of improper linear models in decision making," American Psychologist, vol. 34, no. 7, p. 571-582, 1979.

[25] H. J. Einhorn, "Expert measurement and mechanical combination* 1," Organizational Behavior and Human Performance, vol. 7, no. 1, p. 86106, 1972.

[26] R. Libby, "Man versus model of man: some conflicting evidence* 1," Organizational Behavior and Human Performance, vol. 16, no. 1, p. 112, 1976.

[27] W. H. Wiesner and S. F. Cronshaw, "A meta-analytic investigation of the impact of interview format and degree of structure on the validity of the employment interview," J Occup Psychol, vol. 61, p. 275-90, 1988.

[28] P. E. Meehl, Clinical versus statistical prediction: A theoretical analysis and a review of the evidence. Minneapolis: University of Minnesota Press.

[29] A. R. Damasio, Descartes' error: Emotion, reason, and the human brain. Quill New York:, 2000.

[30] B. Mellers, A. Schwartz, and I. Ritov, "Emotion-based choice," Journal of experimental psychology General, vol. 128, p. 332-345, 1999.

[31] J. R. Harrison and J. G. March, "Decision Making and Postdecision Surprises," Administrative Science Quarterly, vol. 29, no. 1, pp. 26-42, Mar. 1984.

[32] B. Fischhoff, "Hindsight!= foresight: the effect of outcome knowledge on judgment under uncertainty," British Medical Journal, vol. 12, no. 4, p. 304, 2003.

[33] H. Rachlin, Judgment, Decision, and Choice: A Cognitive/Behavioral Synthesis. W.H. Freeman \& Company, 1989.

[34] S. Han, J. S. Lerner, and D. Keltner, "Feelings and consumer decision making: The appraisal-tendency framework," Journal of Consumer Psychology, vol. 17, no. 3, p. 158, 2007 